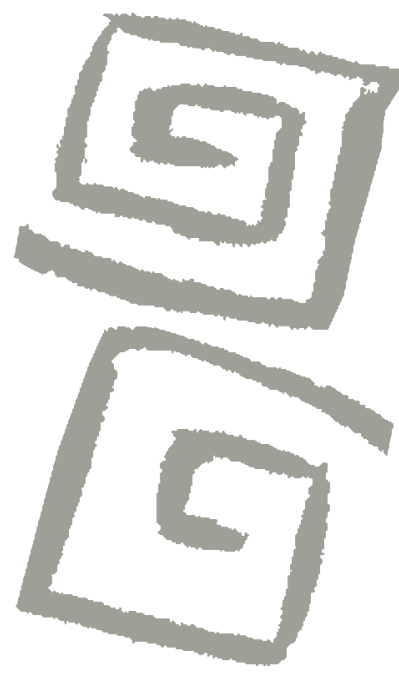

\title{
Salud ocupacional en dos centros de clasificación y acopio de materiales reciclables en el Área Metropolitana de Buenos Aires, Argentina, 2015
}

\author{
Occupational health in two centers for the sorting and \\ collection of recycled materials in the Buenos Aires \\ Metropolitan Area, Argentina, 2015
}

Andrea Mastrangelo ${ }^{1}$, Pablo Schamber ${ }^{2}$

'Doctora en Antropología Social. Coordinadora, Programa Salud, Ambiente y Trabajo, Centro Nacional de Diagnóstico e Investigación en Endemoepidemias, Universidad Nacional de San Martín. Investigadora independiente,

Consejo Nacional de Investigaciones Científicas y Técnicas. Buenos Aires, Argentina. $\triangle$ (iD)

${ }^{2}$ Doctor en Filosofía y Letras. Investigador independiente, Consejo Nacional de Investigaciones Científicas y Técnicas con sede en Universidad Nacional de Quilmes. Profesor, Universidad Nacional de Lanús, Universidad Nacional Arturo Jauretche, Buenos Aires, Argentina. $\triangle$ iD
RESUMEN ¿Cómo estimar los riesgos para la salud de las y los trabajadores que manipulan residuos sólidos urbanos separando y clasificando materiales reciclables? Para responder esta pregunta, durante 2015, se realizó una investigación en terreno con una perspectiva ecoepidemiológica en dos centros de clasificación y acopio (uno urbano y otro en un descampado periurbano) ubicados en la zona sur del Área Metropolitana de Buenos Aires, Argentina. Con el propósito de describir el proceso de trabajo, se utilizaron técnicas etnográficas (entrevistas, observación participante), proyectivas y de trabajo corporal, para caracterizar las condiciones y medio ambiente según riesgos y exigencias de la Organización Internacional de Trabajo para este trabajo no clásico. Las exigencias tenderían a concentrarse en el ingreso y salida de materiales, los riesgos del objeto de trabajo resultarían de la separación y, los riesgos biológicos, del ambiente de trabajo. Se encontró diversidad y poca planificación en el proceso de trabajo. Por ello, la caracterización de las condiciones y el medio ambiente de trabajo fue también una instancia de reflexividad entre trabajadores e investigadores sobre la creación-organización del ambiente de trabajo y la corporización de los padecimientos.

PALABRAS CLAVES Salud Laboral; Residuos; Ambiente; Argentina.

\begin{abstract}
How can the health risks be estimated of workers who handle urban solid waste sorting recyclable materials? To answer this question, during 2015, field research was carried out with an ecoepidemiological perspective in two classification and collection centers (one urban and another peri-urban) located in the southern area of the Metropolitan Area of Buenos Aires, Argentina. In order to describe the work process, ethnographic techniques (interviews, participant observation), as well as other projective and body work techniques were utilized to characterize the conditions and environment of this nonclassical type of work using the framework of risks and demands recommended by the International Labor Organization. The demands tend to concentrate on the entry and exit of materials, the risks of the work object on the sorting of the materials, and the biological risks on the work environment. Within the work process, diversity and little planning were found. Therefore, the characterization of working conditions and environment was also an opportunity for reflexivity among workers and researchers regarding the creation and organization of the work environment and the embodiment of illness.
\end{abstract}

KEY WORDS Occupational Health; Waste Products; Environment; Argentina. 


\section{INTRODUCCIÓN}

El artículo estima los riesgos para la salud de los trabajadores que manipulan residuos sólidos urbanos al clasificar materiales reciclables (papeles, cartones, plásticos, vidrios, metales) en función de las distintas etapas y tareas que involucra el proceso de trabajo del que participan en centros de clasificación y acopio de dichos materiales. Existen antecedentes en el país ${ }^{(1,2)}$ y el exterior ${ }^{(3,4,5,6)}$ que consideraron este aspecto de la práctica de la actividad tanto en la vía pública (recolección en las calles) como en los sitios de disposición de los residuos (basurales a cielo abierto o rellenos sanitarios). No obstante, las condiciones y el medio ambiente de trabajo en centros de clasificación y acopio de materiales reciclables, -ámbito usualmente considerado como una mejora para las condiciones laborales de la actividad- no han sido estudiados.

En Argentina, un informe de la Secretaría de Ambiente y Desarrollo Sustentable de la Nación ${ }^{(7)}$ de 2011 identificó 203 plantas de clasificación de materiales reciclables operativas, el $70 \%$ de las cuales se concentra en la región central del país (provincias de Buenos Aires, Santa Fe y Córdoba). Este informe, que incluyó relevamientos específicos sobre las instalaciones, concluyó que la gestión de las plantas, en general, no está integrada con la etapa de separación de los residuos en origen y la recolección en forma diferenciada, lo que limita las posibilidades de eficiencia y sustentabilidad de los emprendimientos, que dependen de la asignación de subsidios públicos. Si bien el estudio observó que en la organización de las plantas no existía una adecuada división de tareas entre el personal y que las condiciones laborales eran inadecuadas, los aspectos vinculados específicamente a la salud ocupacional y a las condiciones de trabajo no fueron caracterizados en profundidad.

Ante esta situación, en 2015, desde el Programa de Investigación y Desarrollo Tecnológico y Social del Centro Nacional de Diagnóstico e Investigación en Endemoepi- demias de la Administración Nacional de Laboratorios e Institutos de Salud y la Universidad Nacional de San Martín, junto a profesionales de la Universidad Nacional de Lanús que venían analizando los circuitos informales de recuperación de residuos, iniciamos un relevamiento exploratorio que permitió seleccionar dos centros de acopio. Estos centros se constituyeron tanto en lugares de estudio complementarios sobre la diversidad de condiciones y medio ambiente de trabajo, como sobre los distintos tipos de tareas que conforman el contenido del trabajo que allí se realiza, que incluye la promoción de la separación domiciliaria de reciclables y residuos, recolección en vía pública, transporte y descarga en centro de acopio, acarreos internos, cargas y descargas de carros, fardos y bolsones, rotura de bolsas, clasificación por tipos, rotura de botellas de vidrio, aplastado de plásticos y metales y -debido a la desconexión con la separación en origen de residuos y materiales- manipulación de materia orgánica y otros potenciales contaminantes patógenos (con origen en residuos industriales sin clasificar) ${ }^{(8)}$.

En términos teóricos, se consideró a los trabajadores de estos centros como "no clá$\operatorname{sicos}^{\prime \prime(9)}$, pues no son definidos ni por la condición de asalariados, ni por la dependencia del propietario de los medios de producción, sino que son ellos mismos quienes generan los puestos de trabajo -se autoemplean-, negocian el valor de los bienes y crean el mercado donde los obtienen y comercializan. Entender esta práctica como parte de la dinámica social del empleo, implica reconocer un tipo de "sujeto laboral ampliado"(9) pues el trabajador depende de otras inserciones institucionales simultáneas (las redes sociales de las cooperativas analizadas) para la producción y la reproducción social de su rol de trabajador.

\section{METODOLOGÍA}

Este artículo presenta parte de los resultados de un estudio descriptivo interdisciplinario ${ }^{(8)}$ 
de corte ecoepidemiológico ${ }^{(10)}$ más amplio. El equipo de investigación estuvo integrado por antropólogos sociales, parasitólogos, entomólogo, virólogos, fotógrafas y eutonista. En un artículo ya publicado ${ }^{(11)}$ y un capítulo de libro aún en prensa se desarrollaron otros resultados de esa investigación interdisciplinaria, principalmente los vinculados a las exigencias y riesgos biológicos (por ejemplo, presencia de vectores y parásitos) y al análisis de la historia institucional y modalidad organizativa de las cooperativas que gestionan esos centros $^{(12)}$. Complementariamente, en este artículo se privilegia la presentación de los resultados obtenidos con el empleo de metodología etnográfica (entrevistas, observación participante), grupos focales, trabajo corporal y técnicas proyectivas, referidos a los riesgos del trabajo para quienes se desempeñan en las distintas tareas de los mencionados centros de clasificación y acopio.

\section{Los casos}

Se diseñó una muestra no probabilística de selección intencional compuesta por dos centros de clasificación y acopio con características complementarias en lo ambiental (uno nítidamente urbano, y otro en un descampado periférico), la tecnología disponible (con y sin cinta de clasificación, enfardadoras y vehículos propios) y relaciones sociales de producción ("cooperativas" de diferente origen y modalidad organizativa). Ambos casos tienen en común recibir residuos domiciliarios reciclables que idealmente deberían llegar "limpios y secos" dado que son recolectados de modo diferenciado en el marco de programas municipales conocidos como "Bolsa Verde". A través de estos programas los municipios promueven la separación domiciliaria de residuos reciclables, los que deben ser colocados por los vecinos en bolsas de color verde, y establecen días y horarios específicos para su recogida por vehículos propios o contratados a ese efecto.

El centro ubicado en el ámbito urbano es un galpón semiderrumbado cuya superficie mayormente cubierta es de $30 \times 48$ metros, cedido por el municipio a la única iniciativa de recolección diferenciada existente en todo el distrito. La "Bolsa Verde" en este caso es recolectada por miembros de la organización exclusivamente en una localidad residencial de medianos ingresos, aledaña al lugar de estudio. Durante el trabajo de campo documentamos que este centro de acopio recibe también donaciones espontáneas de materiales provenientes de establecimientos (oficinas, comercios e industrias) medianos y pequeños, públicos y privados de otras zonas del municipio.

El centro periurbano en cambio, es un predio alquilado de aproximadamente $10.000 \mathrm{~m}^{2}$ en el que se ubica un galpón de $30 \times 50$ metros. De este modo, algunas tareas de clasificación se realizan bajo techo mientras que otras tienen lugar a la intemperie. Ubicado en un entorno descampado, allí se descarga parte de la recolección selectiva de la campaña "Bolsa Verde" que abarca toda la ciudad. Este lugar recibe también residuos de terceros: algunos como donaciones (industrias, comercios); otros, merced a normativas provinciales implementadas en 2013 que permiten cobrar a los grandes generadores (en este caso barrios privados) por el servicio de transporte y tratamiento (Resoluciones $N^{\circ} 137,138$ y 139 del Organismo Provincial para el Desarrollo Sostenible de la provincia de Buenos Aires).

\section{Las técnicas en campo y el análisis}

Durante 2015 se realizaron ocho visitas sucesivas distribuidas estacionalmente. Mediante observación participante y entrevistas abiertas inicialmente y semiestructuradas después, se caracterizó el proceso de trabajo de cada planta de clasificación y acopio, y se determinaron etapas, tareas y puestos de trabajo. Todas las entrevistas y actividades de campo tuvieron lugar en los centros de acopio, en los horarios de actividad o durante las pausas de higiene y alimentación. Para el relevamiento de las variables de condiciones y medio ambiente de trabajo se instrumentó un diseño metodológico basado en recomendaciones 
de la Organización Internacional del Trabajo $(\mathrm{OIT})^{(13,14)}$, y se distinguieron situaciones externas e independientes de los trabajadores, que constituyen los riesgos, e internas a ellos, que constituyen las exigencias. Los riesgos pueden surgir de los medios de trabajo (luz, ruido, vibraciones, humedad, calor), de los objetos de trabajo (polvos, sustancias químicas y biológicas -se incluyen aquí vectores y reservorios-), pueden generarlos los medios de trabajo en sí mismos (máquinas, herramientas e instalaciones del centro laboral) o derivan del ambiente natural. En cuanto a las exigencias se clasifican en cargas físicas estáticas o posturales, posturas incómodas y forzadas, actividad física dinámica (que incluye esfuerzo físico, desplazamiento, movimientos repetitivos) y organización y división del trabajo (jornadas prolongadas, remuneración por cantidad, modo de gestión, contenido del trabajo).

El criterio de selección de los entrevistados fue que trabajaran en alguno de los dos centros de clasificación y acopio. Sobre la base de los testimonios de todos los trabajadores $(n=40)$ de los dos lugares de estudio, mediante observación participante del proceso de trabajo y grupos focales -en los que se implementaron técnicas proyectivas gráficas (escultura) y corporales (eutonía)-, se describió el contenido del trabajo de cada planta de clasificación dividiéndolo en etapas, tareas y puestos, y definiendo riesgos y exigencias a nivel de los puestos de trabajo.

Para lograr que el relato de los padecimientos producidos en el trabajo tuviera en cuenta las trayectorias de vida de cada trabajador/a, durante tres encuentros, se aplicaron dos técnicas proyectivas a las y los trabajadores: la escultura con identificación de puntos de tensión-placer y la datación de síntomas sobre una silueta humana ${ }^{(15)}$. En esta actividad grupal se propició la corporización ${ }^{(16)}$ mediante ejercicios de eutonía ${ }^{(17)}$. Las técnicas proyectivas con trabajo corporal tuvieron lugar en grupos focales entre trabajadores de la misma etapa y con entrevista en profundidad en el caso que la etapa estuviese a cargo de un/a único/a trabajador/a. Se generaron esculturas de papel de aproximadamente
$25 \mathrm{~cm}$ sobre las que las y los trabajadores realizaron marcas de colores, líneas y fechas señalando cómo estaba el cuerpo antes, durante y después del trabajo. Otro conjunto de datos surgió cuando las y los trabajadores ponían el cuerpo en posición horizontal sobre el suelo o al recorrer puntos de tensión en la espalda con varillas de caña o una pelotita de tenis entre trabajadoras de la misma etapa (clasificación en cinta), mientras realizaban ejercicios de eutonía. En esta instancia grupal de trabajo corporal con técnicas proyectivas, se contrastaban los testimonios orales individuales obtenidos en las conversaciones previas. El registro de este conjunto de prácticas colectivas de investigación fue la fuente de datos sobre la cual se sistematizaron las exigencias corporales en el trabajo de clasificación y acarreo, por lo que el material de testimonios orales no puede ser citado textualmente, pues es relativo a esas prácticas comunes entre investigadores y sujetos participantes del estudio sobre los puntos de tensión o sobrecarga, construyendo una visión colectiva de las exigencias para el puesto de trabajo desde la experiencia de los mismos trabajadores.

En la Figura 1 puede verse a trabajadores señalando los puntos de tensión y placer para determinar las exigencias del puesto clasificación de pie, mientras que, en la Figura 2, puede verse a trabajadores realizando ejercicios de eutonía.

Por este mismo procedimiento se identificaron "puntos mosquitos" y "puntos ratas", como los lugares en los que, desde la perspectiva del actor, aparecían vectores y reservorios, que fueron luego capturados y analizados con metodologías específicas detalladas en otra publicación ${ }^{(11)}$.

Los registros de campo (audios digitales, fotografías, los registros materiales de las técnicas proyectivas y materiales gráficos provistos por las y los trabajadores) fueron analizados con el software Atlas.ti, basándose en la teoría fundamentada en datos ${ }^{(18)}$ cuyos resultados fueron organizados en la matriz de etapas, tareas y puestos, que propone $\mathrm{OIT}^{(13)}$. 


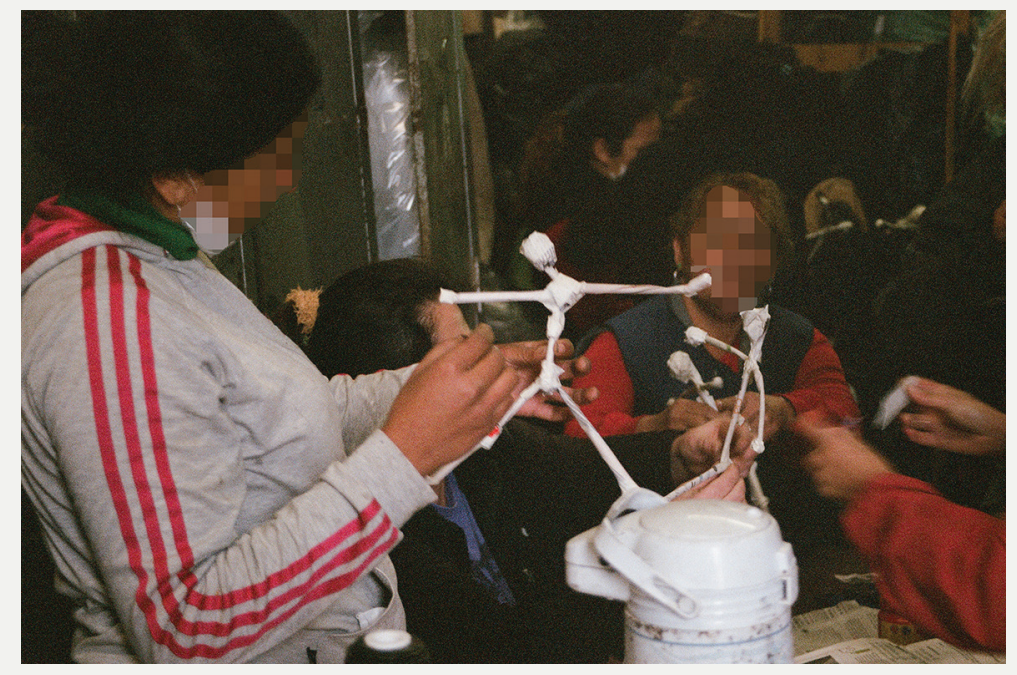

Figura 1. Trabajadores del centro de clasificación y acopio periférico, señalando los puntos de tensión y placer para determinar las exigencias del puesto clasificación de pie. Área Metropolitana de Buenos Aires, Argentina, 2015.

Foto: Pía Leavy.

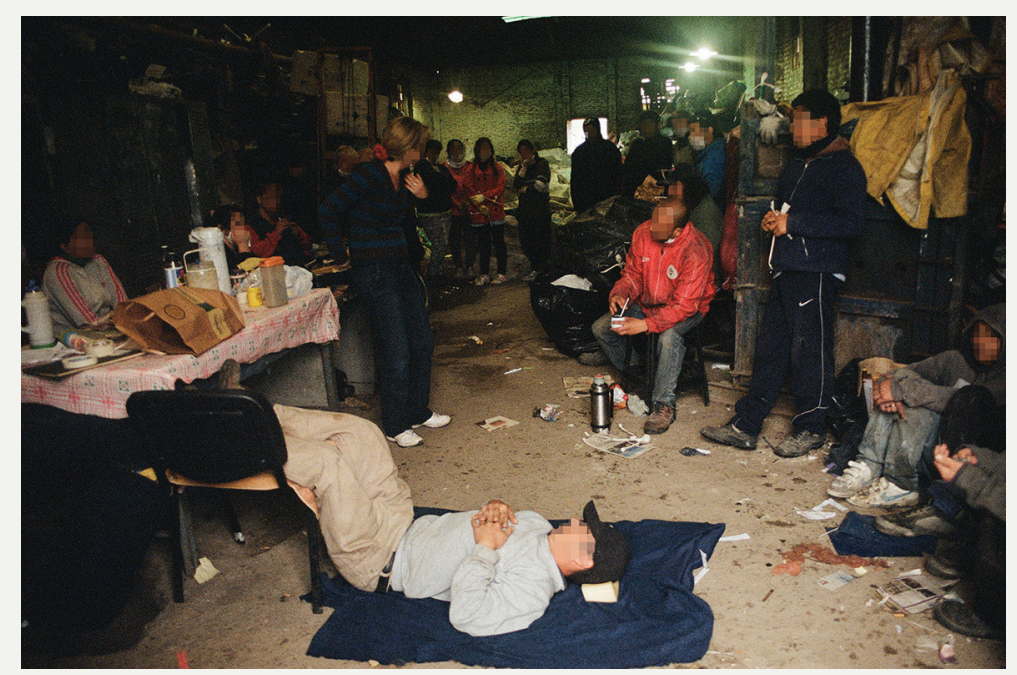

Figura 2. Trabajadores del centro de clasificación y acopio periférico realizando ejercicios de eutonía. Área Metropolitana de Buenos Aires, Argentina, 2015.

Foto: Pía Leavy.

\section{Revisión ética}

El proyecto de investigación fue sometido a revisión bioética y fue aprobado por el Comité de Ética del Instituto Nacional de Enfermedades Virales Humanas “Dr. Julio I.
Maiztegui", Administración Nacional de Laboratorios e Institutos de Salud (INEVH-ANLIS). Se tomó consentimiento informado a los entrevistados, y los investigadores acordaron con los informantes preservar el anonimato de lugares y personas. 


\section{RESULTADOS}

Para determinar riesgos y exigencias fue necesaria la caracterización del proceso de trabajo en los centros de clasificación y acopio seleccionados en la muestra. A pesar de que se trata de una misma figura jurídica ("cooperativa de servicios") que a su vez comparten la identificación social ("cooperativa de cartoneros") ambos emprendimientos productivos se diferencian en su origen, la historia de su conformación, las trayectorias laborales de las y los trabajadores que emplean, la continuidad laboral en la organización, la centralidad de los vínculos de parentesco en la organización del proceso productivo, el modo en que ponderan la antigüedad como jerarquizadora en la organización, el acceso a alimentos o subsidios para distribuir entre los cooperativistas y la mayor o menor injerencia de actores políticos del municipio en su formalización y/o articulación con las autoridades municipales vinculadas con la gestión de residuos, entre otros aspectos. Aun con estas disparidades, estas "cooperativas" son la forma organizativa en la que los municipios del área de estudio delegan la clasificación y el acopio de reciclables.

En el caso del centro urbano, la iniciativa es coordinada por un funcionario municipal con rango de director, quien define la distribución interna de las tareas a cargo de dos grupos claramente diferenciados: uno en horario matutino, cuyas actividades laborales quedan sujetas a las condiciones climáticas (por ejemplo, no se sale a recolectar con lluvia), que se encarga de la promoción de la clasificación domiciliaria y el retiro "puerta a puerta" de los materiales que voluntariamente entregan los vecinos, y otro que realiza durante toda la jornada, dentro del galpón, la descarga y la clasificación manual del contenido de las bolsas retiradas, el acopio de los distintos materiales según su tipo, el traslado y venta de dichos materiales en la sede de un mayorista, y el cálculo de la distribución proporcional de los ingresos obtenidos ( $75 \%$ para ellos y $25 \%$ para el otro grupo). Cabe aclarar que los integrantes del grupo que realiza las tareas de promoción y retiro son beneficiarios del Programa Ingreso Social con Trabajo "Argentina Trabaja", no así los que trabajan en el galpón.

En el caso del centro periurbano, la responsabilidad máxima recae en la presidenta de la organización, quien viene de una trayectoria de tres generaciones de actividad productiva ligada al reciclaje en el seno de su familia (su padre y su abuelo tuvieron lavadero de botellas de vidrio y depósitos dedicados a la compra y venta de materiales reciclables). Ella es, además, la líder de una serie de actividades sociales que se desarroIlan en un local cercano, en el que llevan a cabo acciones de promoción social de distinto tipo (apoyo escolar, planes de estudios para adultos, computación, peluquería, merendero, roperito y biblioteca). El galpón de la cooperativa sirve a la vez como espacio de acopio y distribución de alimentos provistos por distintos estamentos estatales o recuperados de grandes generadores (supermercados). Estos alimentos son entregados como incentivos al presentismo, la puntualidad o el trabajo extra de los trabajadores de este emprendimiento. A diferencia de los trabajadores del centro urbano, los ingresos de los ocupados en el centro periurbano son fijos por mes y no guardan proporcionalidad con las ventas.

Las técnicas proyectivas y corporales, sumadas a la metodología propuesta por la OIT, nos permitieron dilucidar, durante el trabajo de campo, cómo se ordenaba el proceso de trabajo considerando padecimientos previos y cotidianos de las y los trabajadores, sopesando riesgos y exigencias, con conflictos personales, económicos y políticos.

La elaboración de planos de los centros de clasificación y la impresión de las fotografías en las que se observa a operarios/as trabajando o participando en grupos focales, permitieron a las y los trabajadores asociar las etapas del proceso trabajo con espacios dentro del predio y establecer cuáles debían señalizarse, qué modificaciones realizar para evitar accidentes y desgastes, qué protección resultaría necesaria, cómo controlar la reproducción de vectores y reservorios, y cómo 
cubrir los costos que estas acciones demandan. Por ello, al implementar las técnicas de observación y entrevista y los grupos focales se discutieron con las y los trabajadores las recomendaciones de prevención y vigilancia sanitaria. De allí surgió la decisión de confeccionar un calendario del año 2016 con fotos relativas a los dos centros, para venderlo y adquirir medios para el control químico: rodenticidas y larvicidas ${ }^{(19)}$. Asimismo, las y los investigadores continuamos contribuyendo en la elaboración de un sistema de vigilancia de la salud en el trabajo, con libreta sanitaria para la actividad.

La Tabla 1 muestra una síntesis de la organización del trabajo junto con la identificación de riesgos y exigencias para ambos centros. Se trata de un esquema que fue construido y

Tabla 1. Síntesis de exigencias y riesgos para la salud, según etapas y tareas de dos centros de clasificación y acopio (urbano y periférico). Área Metropolitana de Buenos Aires, Argentina, 2015.

\begin{tabular}{|c|c|c|c|c|}
\hline Etapas & Tareas & Exigencias & $\begin{array}{l}\text { Riesgos por objeto de } \\
\text { trabajo }\end{array}$ & Riesgos biológicos \\
\hline Recolección & $\begin{array}{l}\text { Pliegue de bolsas verdes para entregar } \\
\text { a vecinos } \\
\text { Traslado a la localidad y recorrido a pie } \\
\text { "puerta a puerta" entregando bolsas } \\
\text { vacías y retirando bolsas llenas } \\
\text { Ubicación de las bolsas llenas en } \\
\text { extremo de veredas para facilitar } \\
\text { recogida }\end{array}$ & - & $\begin{array}{l}\text { Presencia de materiales } \\
\text { cortantes }\end{array}$ & $\begin{array}{l}\text { Contaminación con heces de gato (reservorio parasitario). Riesgo } \\
\text { por presencia parásitos (reservorio en perros). Presencia de } \\
\text { vectores Aedes aegypti, Culex pipiens }\end{array}$ \\
\hline Carga & Carga de bolsas verdes al camión & Esfuerzo físico & $\begin{array}{l}\text { Seguridad vial por uso de } \\
\text { vehículos deteriorados }\end{array}$ & $\begin{array}{l}\text { Contaminación con heces de gato (reservorio parasitario). Riesgo } \\
\text { por presencia parásitos (reservorio en perros). Presencia de } \\
\text { vectores Aedes aegypti, Culex pipiens }\end{array}$ \\
\hline Traslado & Conducción del vehículo & - & $\begin{array}{l}\text { Seguridad vial por uso de } \\
\text { vehículos deteriorados }\end{array}$ & - \\
\hline Descarga & $\begin{array}{l}\text { Descarga manual de bolsas verdes en } \\
\text { la planta }\end{array}$ & Esfuerzo físico & $\begin{array}{l}\text { Presencia de materiales } \\
\text { cortantes }\end{array}$ & $\begin{array}{l}\text { Contaminación con heces de gato (reservorio parasitario). Riesgo } \\
\text { por presencia parásitos (reservorio en perros). Presencia de } \\
\text { vectores Aedes aegypti, Culex pipiens }\end{array}$ \\
\hline Acarreo & $\begin{array}{l}\text { Carga de zorras y carretas } \\
\text { Traslado de los materiales a los } \\
\text { puestos de trabajo }\end{array}$ & Esfuerzo físico & $\begin{array}{l}\text { Presencia de materiales } \\
\text { cortantes }\end{array}$ & $\begin{array}{l}\text { Contaminación con heces de gato (reservorio parasitario). Riesgo } \\
\text { por presencia parásitos (reservorio en perros). Presencia de } \\
\text { vectores Aedes aegypti, Culex pipiens }\end{array}$ \\
\hline Clasificación & $\begin{array}{l}\text { Descarga de zorras y carretas en } \\
\text { puesto de trabajo } \\
\text { Clasificación de materiales }\end{array}$ & $\begin{array}{l}\text { Poca iluminación. } \\
\text { Fuerza en el tren de } \\
\text { extremidades superiores. } \\
\text { Desgaste asimétrico de } \\
\text { la cintura }\end{array}$ & $\begin{array}{l}\text { Presencia de materiales } \\
\text { cortantes }\end{array}$ & $\begin{array}{l}\text { Contaminación con heces de gato (reservorio parasitario). Riesgo } \\
\text { por presencia parásitos (reservorio en perros). Presencia de } \\
\text { vectores Aedes aegypti, Culex pipiens }\end{array}$ \\
\hline $\begin{array}{l}\text { Embolsado y } \\
\text { enfardado }\end{array}$ & $\begin{array}{l}\text { Traspaso de materiales de pequeñas } \\
\text { bolsas o recipientes a bolsones de } \\
\text { mayor tamaño }\end{array}$ & $\begin{array}{l}\text { Poca iluminación. } \\
\text { Fuerza en el tren de } \\
\text { extremidades superiores. } \\
\text { Desgaste asimétrico de } \\
\text { la cintura }\end{array}$ & $\begin{array}{l}\text { Presencia de materiales } \\
\text { cortantes }\end{array}$ & $\begin{array}{l}\text { Contaminación con heces de gato (reservorio parasitario). Riesgo } \\
\text { por presencia parásitos (reservorio en perros). Presencia de } \\
\text { vectores Aedes aegypti, Culex pipiens }\end{array}$ \\
\hline Acopio & Acarreo de bolsones para acopio & $\begin{array}{l}\text { Esfuerzo físico. Exigencia } \\
\text { en rodillas, trabajadores } \\
\text { metidos en bolsones para } \\
\text { reducir volumen }\end{array}$ & $\begin{array}{l}\text { Presencia de materiales } \\
\text { cortantes }\end{array}$ & $\begin{array}{l}\text { Contaminación con heces de gato (reservorio parasitario). Riesgo } \\
\text { por presencia parásitos (reservorio en perros). Presencia de } \\
\text { vectores Aedes aegypti, Culex pipiens }\end{array}$ \\
\hline Carga & $\begin{array}{l}\text { Acarreo de bolsones hasta camión } \\
\text { Carga manual del camión }\end{array}$ & Esfuerzo físico & $\begin{array}{l}\text { Presencia de materiales } \\
\text { cortantes }\end{array}$ & $\begin{array}{l}\text { Contaminación con heces de gato (reservorio parasitario). Riesgo } \\
\text { por presencia parásitos (reservorio en perros). Presencia de } \\
\text { vectores Aedes aegypti, Culex pipiens }\end{array}$ \\
\hline Traslado & Conducción del vehículo & - & $\begin{array}{l}\text { Seguridad vial por uso de } \\
\text { vehículos deteriorados }\end{array}$ & - \\
\hline Ventas & $\begin{array}{l}\text { Descarga de bolsones en lugar de } \\
\text { venta } \\
\text { Registro de ventas y cálculos } \\
\text { distribución porcentual entre grupos. }\end{array}$ & Esfuerzo físico & $\begin{array}{l}\text { Seguridad vial por uso de } \\
\text { vehículos deteriorados }\end{array}$ & $\begin{array}{l}\text { Contaminación con heces de gato (reservorio parasitario). Riesgo } \\
\text { por presencia parásitos (reservorio en perros). Presencia de } \\
\text { vectores Aedes aegypti, Culex pipiens }\end{array}$ \\
\hline Limpieza & $\begin{array}{l}\text { Barrido } \\
\text { Acopio de material de descarte } \\
\text { Carga de camión con material de } \\
\text { descarte }\end{array}$ & Esfuerzo físico & $\begin{array}{l}\text { Seguridad vial por uso de } \\
\text { vehículos deteriorados }\end{array}$ & $\begin{array}{l}\text { Contaminación con heces de gato (reservorio parasitario). Riesgo } \\
\text { por presencia parásitos (reservorio en perros). Presencia de } \\
\text { vectores Aedes aegypti, Culex pipiens }\end{array}$ \\
\hline
\end{tabular}


consensuado con las y los trabajadores de los centros entre mayo y noviembre 2015.

En síntesis, a pesar de las variables que marcan diferencias estructurales y contingentes de los centros de clasificación y acopio estudiados, concluimos que en ambos casos las exigencias tenderían a concentrarse en las tareas vinculadas al ingreso y salida de materiales, mientras que los riesgos del objeto de trabajo lo harían en la separación y los riesgos biológicos resultarían del ambiente de trabajo.

\section{DISCUSIÓN}

\section{El derecho a la salud en el trabajo no clásico: un debate abierto}

Considerar a estos trabajadores como no clásicos (en vez de categorizarlos como "informales" o "precarios") es una elección teórica ${ }^{(9)}$, ya que entendemos que la situación social en que se insertan no es eventual, ni será superada por la expansión de las fuerzas productivas. Es un trabajo no clásico desarrollado por los propios trabajadores que generaron un servicio, valiéndose simultáneamente de dos oportunidades: por un lado, satisfacer la demanda de materias primas de las industrias a bajo costo, a partir de los descartes domésticos y fabriles y, por otro, ofrecer a la gestión municipal la posibilidad de reducir los volúmenes de basura destinados a entierro, y los gastos relacionados con su traslado y disposición final.

En este estudio no recurrimos a la categorización de "informal", que sí ha sido productiva para otros análisis de este fenómeno multidimensional ${ }^{(20,21)}$, porque independientemente de que se cumpla con leyes laborales, son los trabajadores quienes, con diferentes grados de capitalización, generan el producto, el mercado (contratos públicos o privados) y los puestos de trabajo.

El trabajo no clásico de los centros de clasificación y acopio implica, también, un "sujeto laboral ampliado"(9) que se integra a "subsistemas económicos"(22,23,24), produciendo interacciones físicas o virtuales con otros. En este sentido, es relevante el trabajo de proveedores y clientes para hacer posible el trabajo de los clasificadores. Sin los vecinos que contratan el acarreo (countries y barrios privados) o separan en "Bolsas Verdes" (servicio público de recolección selectiva), sin los generadores industriales que regalan su basura, sin la regulación fiscal de los "destinos sustentables", sin la industria o los intermediarios mayoristas de materiales, el trabajo en los centros de acopio no existiría como tal. La disponibilidad de otras fuentes permanentes o esporádicas de ingreso para los ocupados en los centros de clasificación y acopio, y la posibilidad de retornar a estos, forma parte también de ese escenario laboral ampliado.

Sin embargo, hay un aspecto en que los trabajadores de los centros de acopio son trabajadores clásicos. Con la instalación en los galpones, separaron el domicilio de su propio trabajo, aunque se llevaron consigo los riesgos y exigencias propias de su labor. En el caso de los centros de acopio estudiados, salir de la recolección en la calle y generar "el galpón" para la clasificación y "la cooperativa" como formato jurídico implicó un proceso de organización del proceso de trabajo, alianzas políticas y aportes económicos, a la vez que separó la vida doméstica del trabajo, hecho especialmente relevante porque la mayoría son trabajadoras.

Los numerosos estudios sociales sobre el riesgo y lo laboral abrevan en unas pocas teorías $^{(1,25,26,27,28,29)}$. Considerando que, en el siglo precedente, la principal fuente de reducción de riesgo era la sociedad salarial(1) a través de la cual el Estado reconocía derechos y daba acceso a la seguridad social, cabe preguntarnos si, en la actualidad, en el trabajo no clásico, la consideración del riesgo laboral y la salud ocupacional pueden (o deben en términos del respeto a la igualdad jurídica de las personas) plantearse del mismo modo para el actor social heterogéneo que son las "cooperativas" que clasifican, acopian y comercializan material recuperado de los residuos.

El trabajo de recuperador de materiales, como cualquier otro trabajo, a la vez que genera la seguridad de un ingreso es productor 
de riesgo. En un presente en el que el Estado y la seguridad social solidaria se van limitando, la vulnerabilidad de los no asalariados es aún mayor. De este modo, sostenemos que, en el trabajo en centros de acopio, entre riesgo e ingresos se da una relación inversamente proporcional: los ingresos reducen la incertidumbre, pero el modo de obtenerlos en sujetos socialmente vulnerables hace que la exposición al riesgo sea potencialmente mayor, pues trabajan fuera de todo mecanismo de salvaguarda y con un capital simbólico menor.

Ante este panorama, entendemos que no se trata de imponer una noción de riesgo de los colectivos asalariados a los no clásicos, extrapolando las trayectorias laborales o dejando en suspenso la vulnerabilidad o el riesgo social. Tampoco se trata de lograr una ponderación numérica o estadística del riesgo como la requerida por los seguros. Nuestra propuesta, sobre la base de las metodologías cualitativas implementadas, es tender a una definición transaccional del riesgo, la salud, las condiciones y el medio ambiente de trabajo, reflexionando con los trabajadores sobre las tareas del puesto de trabajo, en la organización en la que participan.

Al estudiar el riesgo y las exigencias junto con la organización del proceso de trabajo, entendemos que no solo la maximización de la productividad es el criterio que rige la organización de las etapas y tareas. La edad y el género, la antigüedad en el grupo, el desgaste preexistente de los trabajadores, las relaciones de parentesco o afinidad, tanto como la donación por parte de actores sociales públicos o privados de cintas transportadoras, básculas o compresores, la disponibilidad de agua o fuerza motriz estructuran la organización y condicionan la seguridad y la productividad.

\section{La relación teoría-metodología en los casos de estudio}

Las intervenciones desde las políticas locales de gestión de residuos hacia los recuperadores urbanos han venido modificándose a lo largo del tiempo ${ }^{(30)}$ y los cambios se aceleraron en los últimos 20 años $^{(31)}$. La práctica laboral de los cartoneros dejó de estar invisibilizada para comenzar a ser valorada positivamente en la agenda de políticas públicas.

Aunque compartan el hecho de ser "cooperativas de cartoneros", como mostramos al caracterizarlas $^{(12)}$, las modalidades organizativas, las formas en que se generan los puestos de trabajo, la contratación y la capitalización varían drásticamente. Es más, puestos a comprender la subjetivación en los procesos colectivos, la categoría jurídica "cooperativa" debería complejizarse en muchas dimensiones para describir relaciones laborales de dependencia encubiertas y jerarquizaciones subalternizantes.

En los estudios ya señalados sobre salud ocupacional en "cartoneros" ${ }^{\text {" }(1,2,3,4,5,6)}$ predomina la presentación de casos con diversas relaciones sociales de producción, a los que se aplican encuestas estructuradas con base en parámetros sobre riesgo y salud laboral en cierta medida estándares, susceptibles de aplicarse mutatis mutandis a cualquier actividad generadora de ingresos. Algunos artículos determinan riesgo únicamente en perspectiva de los trabajadores ${ }^{(4,5)}$ o del sistema de salud pública ${ }^{(1)}$. De haberse aplicado en nuestro estudio metodologías semejantes, las singularidades enunciadas para cada caso, que incluyen particulares trayectorias personales y organizativas que resultan en las condiciones y medio ambiente de trabajo, difícilmente hubiesen podido advertirse.

A diferencia de otros estudios que agrupan a los "cartoneros" en una identidad laboral consensuada y más o menos homogénea $^{(1)}$, consideramos que los grupos de trabajadores estudiados no corresponden a un colectivo identitario específico, por lo que los diferentes polos culturales ${ }^{(29)}$ respecto del riesgo no se asocian con variables externas a las condiciones y el medio ambiente, ni son completamente individuales. Por ello, la metodología implementada se orientó a determinar colectivamente la racionalidad productiva y organizativa endógena al proceso de trabajo, acotando los riesgos y exigencias que de él emanan. En los estudios de caso 
presentados, el trabajo etnográfico, junto con las técnicas proyectivas, las fotografías y el trabajo corporal fueron centrales para construir conocimiento reflexionando junto con los trabajadores. Consideramos que el involucramiento de los trabajadores en la corporización ${ }^{(16)}$ de los padecimientos ${ }^{(32)}$ asociados a los puestos de trabajo, resulta también central para pensar la prevención, ya que recae en manos de las mismas organizaciones de trabajadores y requiere de su protagonismo.

Fue con esta intención que transformamos la herramienta un tanto tipológica y prescriptiva de la $\mathrm{OIT}^{(13,14)}$ en un instrumento de reflexión en los grupos focales con técnicas proyectivas y corporales compartidas con los trabajadores. Así, mientras recopilábamos información sobre el proceso de trabajo, las entrevistas daban cuenta de los tenues equilibrios logrados en la organización del proceso productivo, las jerarquías productivas, políticas y de género, la alternancia de trabajadores entre los puestos, las exigencias que generan dolor y enfermedad, tanto como la convivencia con animales domésticos y sinantrópicos que eventualmente eran reservorios de enfermedad y vectores de virus y parásitos ${ }^{(11,12)}$. Por ello, consideramos que el diseño metodológico interdisciplinario presentado, es útil a la comprensión de la salud ocupacional en el trabajo no clásico y puede replicarse en otros estudios.

\section{AGRADECIMIENTOS}

Fueron responsables de la investigación sobre riesgo biológico Arturo Lizuain y María Soledad Santini (CeNDIE-ANLIS) investigación entomológica, Natalia Guerreiro Martins y Rosario Robles (CEPAVE-CONICET-UNLP) investigación parasitológica en roedores y heces de caninos y felinos, Victorio Palacio y Silvina Goenaga (INEVH-ANLIS) virología, Verónica Eggers (Trabajo Saludable) fue la eutonista, Pia Leavy (CONICET-UBA) y Laura Díaz Galán (Atlas social) fueron las fotógrafas.

La investigación fue financiada por la Beca "Carrillo-Oñativia" en categoría de estudio colaborativo multicéntrico, otorgada por la Dirección de Investigación para la Salud, Ministerio de Salud de la Nación, Argentina, y registrada bajo el número IS000925 en el Registro Nacional de Investigaciones en Salud (RENIS) del Sistema Integrado de Información Sanitaria Argentina. Andrea Mastrangelo fue la coordinadora del estudio.

\section{REFERENCIAS BIBLIOGRÁFICAS}

1. Panaia M. Accidentes de trabajo en el "cartoneo". Identidad y medioambiente. En: Panaia M. Sociología del riesgo accidentes de trabajo en el sector informal. Buenos Aires: Miño y Dávila; 2008.

2. Parizeau K. Un estudio de la Salud de los Cartoneros de Buenos Aires: Peligro y desigualdad. En: Suárez F, Schamber P. Recicloscopio II: Miradas sobre recuperadores urbanos de América Latina. Buenos Aires: Ciccus; 2011.

3. Gómez-Correa Jaime A, Agudelo-Suárez Andrés A, Ronda-Pérez Elena. Condiciones sociales y de salud de los recicladores de Medellín. Revista de Salud Pública. 2008;10(5):706-715.

4. Velloso MP, Guimaraes MBL. A imagem na pesquisa qualitativa em saúde. Ciéncia \& Saúde Coletiva. 2013;18(1):245-252. 
5. Cavalcante S, Franco MFA. Profissão perigo: percepção de risco à saúde entre os catadores do Lixao do Jangurussu. Revista Subjetividades. 2007;7(1):211-231.

6. Castilhos Junior AB, Ramos NF, Alves CM, Forcellini FA, Graciolli OD. Catadores de materiais recicláveis: análise das condições de trabalho e infraestrutura operacional no Sul, Sudeste e Nordeste do Brasil. Ciência \& Saúde Coletiva. 2013;18(11):3115-3124.

7. Grupo Arrayanes. Proyecto nacional para la gestión integral de residuos sólidos urbanos BIRF 7362-AR [Internet]. Buenos Aires: Observatorio Nacional de Gestión de Residuos Sólidos Urbanos; 2011 [citado 6 mar 2019]. Disponible en: https:// tinyurl.com/y6evqwdn.

8. Mastrangelo A, Schamber PJ, Lizuain AA, Guerreiro Martins $\mathrm{N}$, Palacio $\mathrm{V}$. Descripción y análisis de la percepción y exposición a riesgo de enfermedades zoonóticas en trabajadores de plantas cooperativas de acopio y separación de residuos para reciclado en el Área Metropolitana de Buenos Aires. Buenos Aires: Registro Nacional de Investigaciones en Salud (IS000925); 2015.

9. De la Garza Toledo E. Trabajo no clásico y flexibilidad. Caderno SRH. 2013;26(68):315-330.

10. Susser M, Susser E. Choosing a future for epidemiology: I Eras and paradigms. American Journal of Public Health. 1996;86(5):668-673.

11. Mastrangelo AV, Schamber PJ, Lizuain AA, Guerreiro Martins N, Palacio V. Estudio descriptivo sobre salud ocupacional en centros de clasificación de material reciclable en el área metropolitana de Buenos Aires. Revista Argentina de Salud Pública. 2016;7(Número especial):13.

12. Mastrangelo AV, Schamber PJ, Lizuain AA, Guerreiro Martins N, Palacio V. Salud y trabajo en centros de acopio de material reciclable del AMBA: Exploración interdisciplinaria a partir del estudio descriptivo de dos casos. En: Schamber PJ, Suárez F. Recicloscopio VI. Buenos Aires: UNGSUNLa-CICCUS. (En prensa, 2019).

13. Organización Internacional del Trabajo. Las condiciones y medio ambiente de trabajo para los facilitadores [Internet]. Buenos Aires: OIT; 2003 [citado 29 mar 2016]. Disponible en: https:// tinyurl.com/yay4sto3.

14. Organización Internacional del Trabajo. La salud y la seguridad en el trabajo (series) [Internet]. Buenos Aires: OIT; 1996 [citado 29 mar 2016]. Disponible en: https://tinyurl.com/yy5ouf8u.
15. López Barberá E. La escultura y otras técnicas proyectivas aplicadas en psicoterapia. Barcelona: Paidós; 1997.

16. Citro S. La antropología del cuerpo y los cuerpos en-el-mundo: Indicios para una genealogía (in)disciplinar. En: Citro S. Cuerpos Plurales: Antropología de y desde los cuerpos. Buenos Aires: Biblos; 2011.

17. Rovella A. La eutonía: Sus principios. Jornadas de Cuerpo y Cultura, Universidad Nacional de La Plata [Internet]. 2008 [citado 29 mar 2016]. Disponible en: https://tinyurl.com/yagqztze.

18. Glaser B, Strauss A. The discovery of grounded theory: strategies for qualitative research. New York: Aldine Publishing; 1967.

19. Leavy P, Díaz Galán L. Calendario 2016, sobre el trabajo en los centros de acopio del Estudio colaborativo multicéntrico salud ocupacional en centros de acopio de reciclable. Buenos Aires: PDTS UNAJ, UNLa, UNQ, CONICET, UNSAMCeNDIE ANLIS; 2015.

20. Saraví G. Detrás de la basura: cirujas; Notas sobre el sector informal urbano. En: Quirós G, Saraví G. La informalidad económica, ensayos de antropología urbana. Buenos Aires: CEAL; 1994.

21. Schamber P, Suárez F. El cirujeo y la gestión de los residuos: Una mirada sobre el circuito informal del reciclaje en el conurbano bonaerense. Revista Realidad Económica. 2002;190:32-63.

22. Lacabana M, Schamber P, Moreno F. Subsistemas económicos, territorio y ambiente: $\mathrm{El}$ reciclaje en el conurbano sur de Buenos Aires. Proyección. 2015;IX(18):118-145.

23. Schamber P, Lacabana M, Moreno F. De convertidor industrial a depositero polirubro: Trayectoria y actividad productiva de un acopiador de residuos reciclables de Quilmes. Revista de Ciencias Sociales, segunda época. 2015;7(27):139-161.

24. Schamber $P$, Lacabana M, Bressano C. Detritus del consumo y producción industrial en el territorio del reciclaje: Análisis de la intermediación en el sur del conurbano bonaerense. Revista Iberoamericana de Estudios Municipales (RIEM). 2015; VI(11):120-143.

25. Douglas M. La aceptabilidad del riesgo según las ciencias sociales. Barcelona: Paidós; 1996.

26. Ewald F. L'Etat providence. Paris: Grasset; 1986.

27. Beck U. La sociedad del riesgo: Hacia una nueva modernidad. Barcelona: Paidós; 2000. 
28. Giddens A. Consecuencias de la modernidad. Madrid: Alianza Editorial; 2001.

29. Douglas M, Wildavsky J. Risk and culture. Oakland: University of California Press; 1984.

30. Suárez F. La Reina del Plata, Buenos Aires: sociedad y residuos. Los Polvorines: Ediciones Universidad Nacional de General Sarmiento; 2016.
31. Dimarco S. Entre el trabajo y la basura: sociohistoria de la clasificación de residuos en la Ciudad de Buenos Aires (1870-2005). [Tesis de Doctorado en Ciencias Sociales]. Buenos Aires: UBA; 2010.

32. Fassin D. Gobernar por los cuerpos, políticas de reconocimiento hacia los pobres y los inmigrantes en Francia. Cuadernos de Antropología Social. 2003;(17):49-78.

FORMA DE CITAR

Mastrangelo A, Schamber P. Salud ocupacional en dos centros de clasificación y acopio de materiales reciclables en el Área Metropolitana de Buenos Aires, Argentina, 2015. Salud Colectiva. 2019;15:e1348. doi: 10.18294/sc.2019.1348.

Recibido: 13 de marzo de 2017 | Versión final: 20 de diciembre de 2017 | Aprobado: 29 de enero de 2018

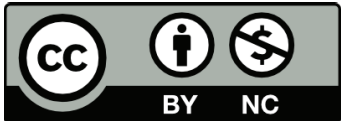

Esta obra está bajo una licencia de Creative Commons Reconocimiento-NoComercial 4.0 Internacional. Reconocimiento - Permite copiar, distribuir y comunicar públicamente la obra. A cambio, se debe reconocer y citar al autor original. No Comercial - Esta obra no puede ser utilizada con finalidades comerciales, a menos que se obtenga el permiso. 